\title{
Experimental tests of the trigger prototype for the AMADEUS experiment based on Sci-Fi read by MPPC
}

\author{
M. Bazzi, C. Berucci, G. Corradi, C. Curceanu, A. D’Uffizi, K. Piscicchia, M. Poli Lener, A. Rizzo, \\ A. Romero Vidal, E. Sbardella, A. Scordo*, D. Tagnani, O. Vazquez Doce \\ Laboratori Nazionali di Frascati, INFN, via E. Fermi 40, 00044 Frascati (RM), Italy
}

\section{A R T I C L E I N F O}

\section{Article history:}

Received 15 September 2011

Received in revised form

23 December 2011

Accepted 26 December 2011

Available online 30 December 2011

\section{Keywords:}

MPPC

Scintillating fibers

AMADEUS experiment

Trigger for kaons

\begin{abstract}
A B S T R A C T
The Multi-Pixel Photon Counter (MPPC) detectors consist of hundreds of micro silicon Avalanche PhotoDiodes (APD) working in Geiger mode. The high gain and the small dimensions typical of these devices, together with their good performances in magnetic field, make them ideal readout devices for scintillating fibers as trigger detectors in particle and nuclear physics experiments like AMADEUS, where such a system is planned to be used to trigger on charged kaons. A prototype setup for this trigger system, consisting of 5 scintillating fibers readout by 10 MPPCs, was built and tested in laboratory and mounted inside the DAФNE collider at LNF-Frascati to measure the back-to-back $\mathrm{K}^{+} \mathrm{K}^{-}$ pairs emitted in the $\Phi$-decay processes. The ad hoc readout electronics was designed and realized at Laboratori Nazionali di Frascati (INFN). A 64 channels setup, with a new dedicated electronics, was then built and characterized in the laboratory. The results of the tests are presented and discussed.
\end{abstract}

(c) 2012 Elsevier B.V. All rights reserved.

\section{Introduction}

The AMADEUS experiment [1] aims to perform measurements of low-energy charged kaons interactions in nuclear matter, in particular to search for the so-called "kaonic nuclear clusters". The AMADEUS setup is going to be installed inside the KLOE detector [2] in the free space inside the drift chamber. The experiment will then use the drift chamber and the calorimeter of the KLOE detector, together with a dedicated setup consisting of a target cell to be filled with deuterium or helium (3 and 4) and a trigger system, which will trigger on the back-to-back $\mathrm{K}^{+} \mathrm{K}^{-}$pairs emitted from the decays of the $\Phi$ particles produced at the DAФNE $\mathrm{e}^{+} \mathrm{e}^{-}$collider of LNF-INFN [3,4]. There are very strict constraints for the trigger system, such as a very limited space and necessity to operate the detectors in magnetic field. In addition to this, a timing resolution of few hundreds of ps is needed, in order to separate by Time of Flight (TOF) the kaons, coming from the DAФNE machine, from all other background particles, mostly of electromagnetic type, generated by electrons and positrons lost from the circulating beams. Ideal for such a role are the Multi Pixel Photon Counter detectors (MPPC) [5-8], coupled to scintillating fibers (Sci-Fi). MPPC were tested in laboratory, with an ad hoc built electronics. Then, a prototype of $10 \mathrm{MPPC}$ reading $5 \mathrm{Sci}-\mathrm{Fi}$ was built and tested inside DAФNE. A second prototype of 64 channels has then been built and is under characterization.

\footnotetext{
* Corresponding author. Tel.: + 390694038447.

E-mail address: alessandro.scordo@lnf.infn.it (A. Scordo).
}

\section{MPPC selection and readout electronics}

The choice of the MPPC device type is a crucial item, and has to be done taking into account a series of parameters as: the number of pixels, the fill factor, the peak sensitivity wavelength $(400 \mathrm{~nm}$ in our case), the photon detection efficiency, the dark current rate and the gain. After having performed tests on MPPC devices from different producers, we checked that the one which matches at best our requirements is HAMAMATSU S10362-11-050U, equipped with metallic package. This device has $1 \mathrm{~mm}^{2}$ of active area, 400 pixels $50 \times 50 \mu \mathrm{m}^{2}$ each, and a gain factor (at room temperature) of $7.5 \times 10^{5}$.

Another key issue to be dealt with in designing and realizing the AMADEUS trigger prototype is the MPPC readout electronics. A dedicated electronic module was designed and built at LNF for the MPPC readout. It consists of two 5 channels NIM modules, providing the $\mathrm{HV}$ for 10 preamplifier boards; the high voltages are individually selectable up to $75 \mathrm{~V}$ with a precision of $0.01 \mathrm{~V}$; the amplifier gives an adjustable 50/100 amplification factor. Each input analog signal is also processed by a discriminator, which allows a threshold from $-0.1 \mathrm{mV}$ to $-200 \mathrm{mV}$ and gives a NIM output signal of 120 ns width.

\section{MPPC laboratory tests with a beta source}

Working at room temperature, thermal excitation could trigger the activation of some pixels (dark current). The MPPCs used 
in this work show a d.c. corresponding to a signal of few activated pixels (a.p.) with a rate in the range of $10^{5} \mathrm{~Hz}$; an accurate study of this dark current is then needed in order to characterize such detectors and their compatibility with the AMADEUS environment. A first set of measurements with scintillating fibers were performed in laboratory. Round shaped ( $1 \mathrm{~mm}$ diameter), $20 \mathrm{~cm}$ long Kuraray SCSF-81 fibers were used, activated by a Sr90 beta source, delivering 0.546 and $2.274 \mathrm{MeV} / \mathrm{c}$ momentum electrons; characteristic constants of SCSF-81 are shown in Table 1 . In order to investigate the dark current, measurements with and without the beta source were performed; frequency of the signals were measured using a scaler module for different thresholds. Results are shown in Fig. 1.

As one can see from Fig. 1, setting the threshold at 4 a.p. peak the dark current contribution becomes negligible; no cooling in order to reduce d.c. is then needed if signals higher than four photons are expected. Triggering with one MPPC with a threshold above 4 a.p., the signal from the other MPPC can be acquired and the resulting spectra are shown in Fig. 2.

According to Monte Carlo simulations, kaons coming from DAФNE are expected to release in the fibers almost a factor 10 more energy than the electrons coming both from the Sr90 source and from the background inside the collider; therefore, no cooling is needed for the dark count rate reduction in our range of interest. In Table 2 results of the Monte Carlo simulations are shown.

Table 1

Kuraray SCSF-81 and Bicron BCF-10 specifications.

\begin{tabular}{lll}
\hline Fiber characteristic & SCSF-81 [9] & BCF-10 [10] \\
\hline Decay time (ns) & 2.4 & 2.7 \\
Attenuation length (m) & 3.2 & 2.2 \\
Trapping efficiency (\%) & 4 & $\geq 5.6$ \\
\hline
\end{tabular}

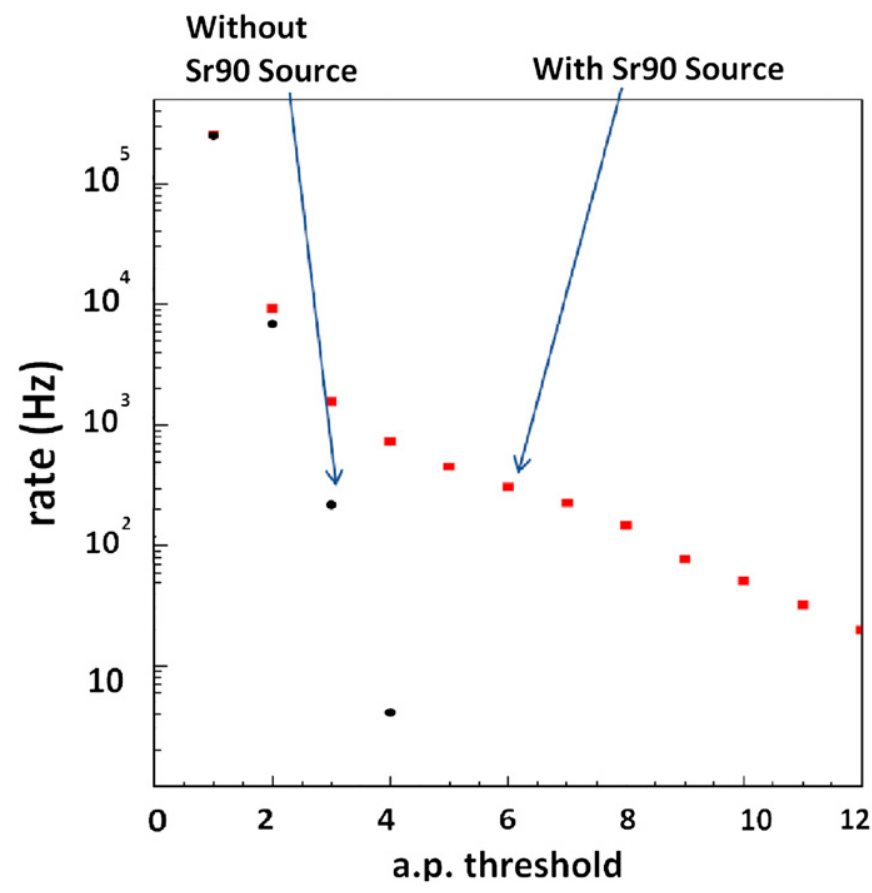

Fig. 1. Rates of the events with and without Sr90 source for different thresholds

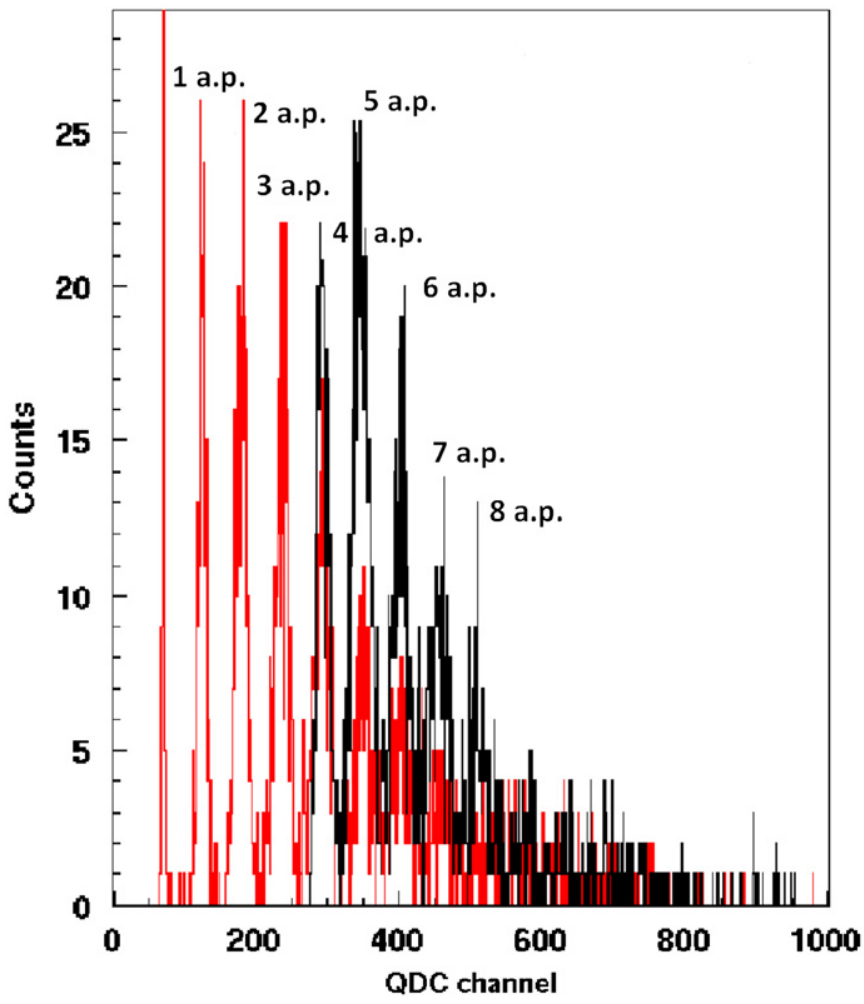

Fig. 2. Spectra from both sides of the fiber and threshold at 4 a.p.; the black spectrum corresponds to the triggering MPPC while the red one corresponds to triggered MPPC. (For interpretation of the references to color in this figure legend, the reader is referred to the web version of this article.)

Table 2

Energy loss by Sr90 and DAФNE electrons and kaons in a $1 \mathrm{~mm}$ thick scintillating fiber (Monte Carlo simulation)

\begin{tabular}{lcl}
\hline Particles & Momentum $(\mathrm{MeV} / c)$ & Energy loss $(\mathrm{MeV})$ \\
\hline Sr90 $\mathrm{e}^{-}$ & 0.546 & 0.27 \\
$\mathrm{Sr90} \mathrm{e}^{-}$ & 2.274 & 0.19 \\
DAФNE e & $\simeq 500$ & 0.32 \\
DAФNE K & 127 & 1.94 \\
\hline
\end{tabular}

\section{The AMADEUS trigger prototype and detection of kaons in DAФNE}

A prototype, consisting of five scintillating fibers coupled at both ends with MPPCs and the readout electronics, was built and tested on the DAФNE collider at Laboratori Nazionali di Frascati (INFN), in the interaction region of the SIDDHARTA experiment [11]. Round shaped ( $1 \mathrm{~mm}$ diameter), $35 \mathrm{~cm}$ long Bicron BCF-10 fibers were used in this setup, whose main characteristic are summarized in Table 1.

The setup was installed below the lower scintillator of the SIDDHARTA Kaon Monitor (KM), which consists of two scintillators one above and one below the beam pipe, as shown in Fig. 3. The TDC signals of the SIDDHARTA KM were included in the data acquisition.

The SIDDHARTA Kaon Monitor represents a good tagger for kaons and a good timing reference in order to test the real triggering capabilities of the AMADEUS trigger prototype.

Since the acquisition chain was driven by the SIDDHARTA KM, only events in which a signal is collected in the scintillators were acquired. In the TDC spectra, a double structure of peaks is clearly visible (see Fig. 4); this double structure is due to the fact that signals coming from two subsequent collisions were acquired in 


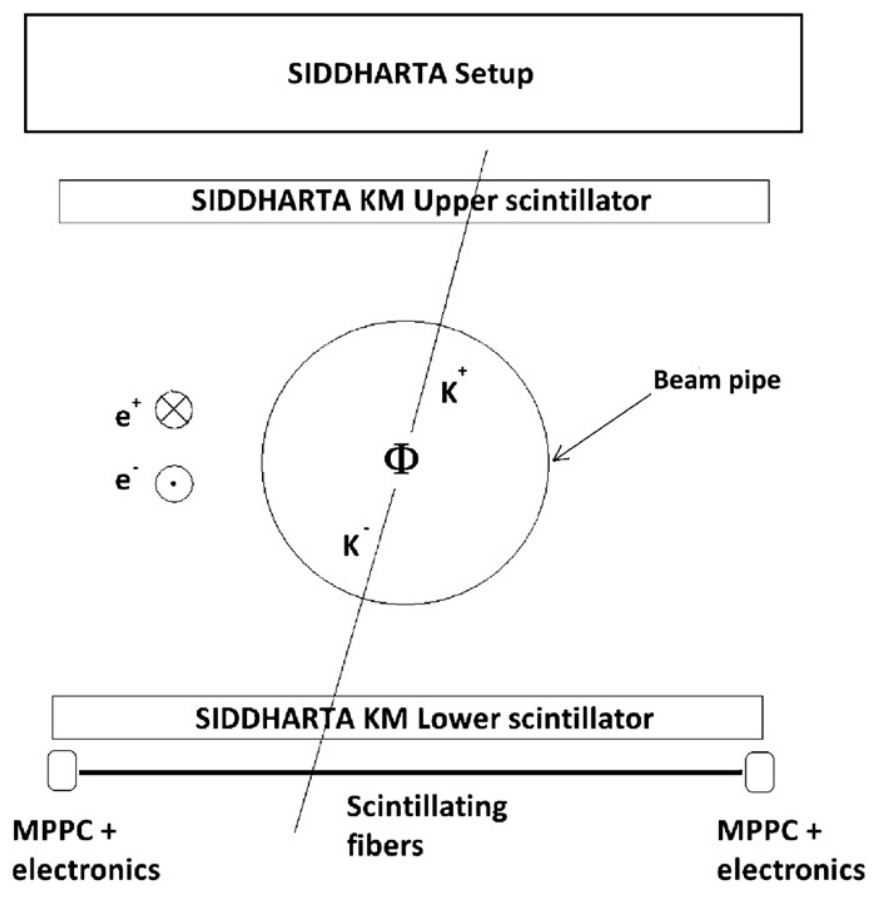

Fig. 3. Schematic transversal view of the prototype setup mounted on the DAФNE collider during the SIDDHARTA experiment.

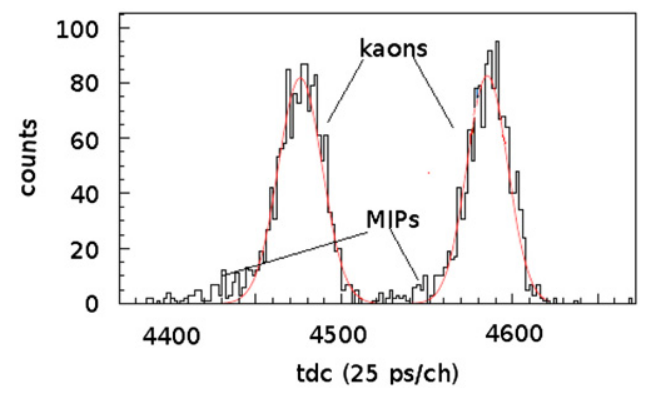

Fig. 4. MPPC TDC spectrum of the AMADEUS trigger prototype. The red superimposed line is the Gaussian fit of the peaks. (For interpretation of the references to color in this figure legend, the reader is referred to the web version of this article.)

the same timing window. Half the radio frequency of the DAФNE machine was used as precise clock for the measurements. The time distance between the two structures is $\simeq 3 \mathrm{~ns}(\mathrm{RF}=365 \mathrm{MHz})$ while the measured time resolution $(\sigma)$ of Sci-Fi plus MPPC setup is $\simeq 300 \mathrm{ps}$.

The ADC spectra of the MPPCs have also been collected and are shown in Fig. 5. A selection of the events have been performed using the information coming from the Kaon Monitor; the overall $\mathrm{ADC}$ spectrum (black, with no request on $\mathrm{KM}$ ) and spectra obtained selecting kaons (green), MIP electrons (blue) or both (red) could then be observed.

\section{A new 64 channels trigger prototype}

Based on the success of first tests, a new and bigger setup has been realized, in order to optimize the geometry and to study the possibility of using Sci-Fi and MPPCs both as trigger and as tracking systems. The setup consists of two separate metallic rings hosting 32 scintillating fibers read by 64 Hamamatsu S10362-11-050U MPPC organized in four independent layers of eight fibers each. The angle between the layers can be changed.

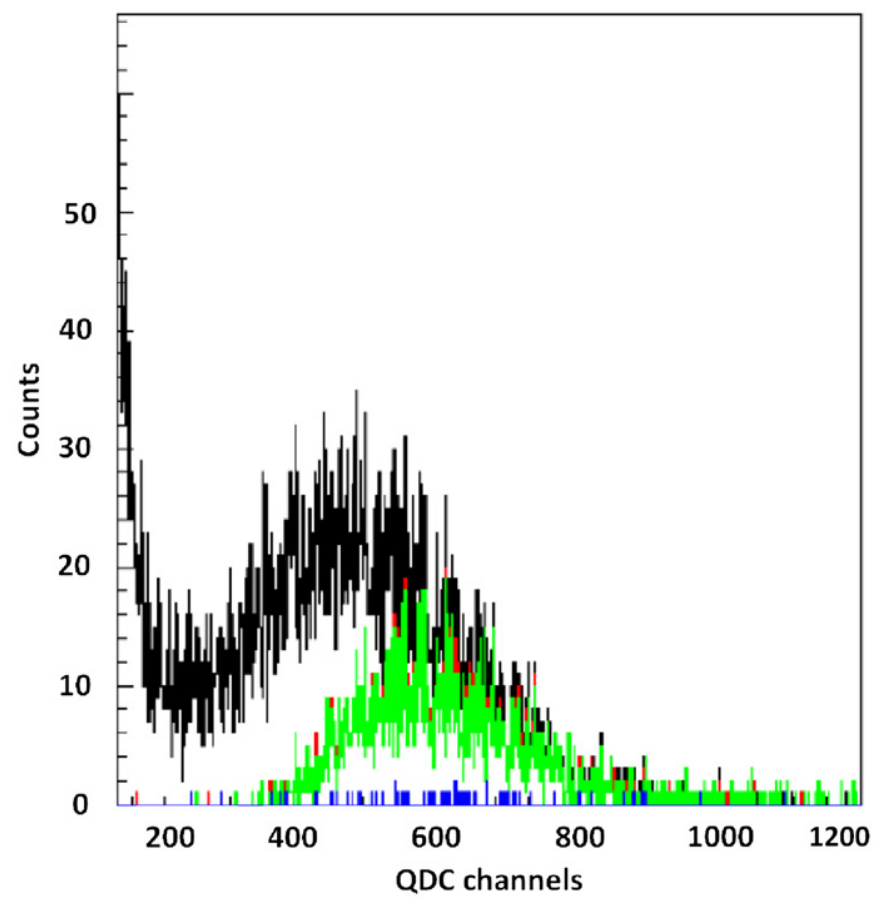

Fig. 5. ADC spectra for MPPCs with different selections on the KM; total spectrum (black) is shown together with cut spectra corresponding to all events (red) on the KM, kaons events (green) or MIPs events (blue). (For interpretation of the references to color in this figure legend, the reader is referred to the web version of this article.)

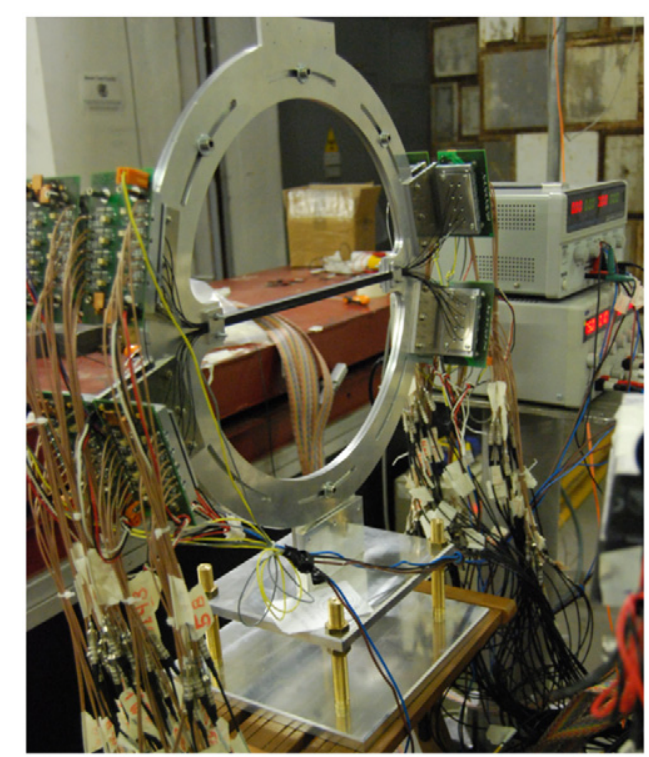

Fig. 6. The 64 channels prototype consisting in two rings, each of them formed by a double layer of eight fibers each; each fiber is read at both ends.

A picture of the setup is shown in Fig. 6. Each fiber is read at both ends; MPPC signals are pre-amplified in a eight-channel board developed at the LNF, providing $a \simeq 10$ amplification factor. Signals are singly processed by a constant fraction discriminator module (also developed at LNF) which provides 64 ECL outputs and 5 NIM signals corresponding to the logic OR of the 64 channels.

Performances of the preamplifiers and the time resolution have been checked for the new electronics, in laboratory tests in which the light of a blue laser was sent directly on the device's 


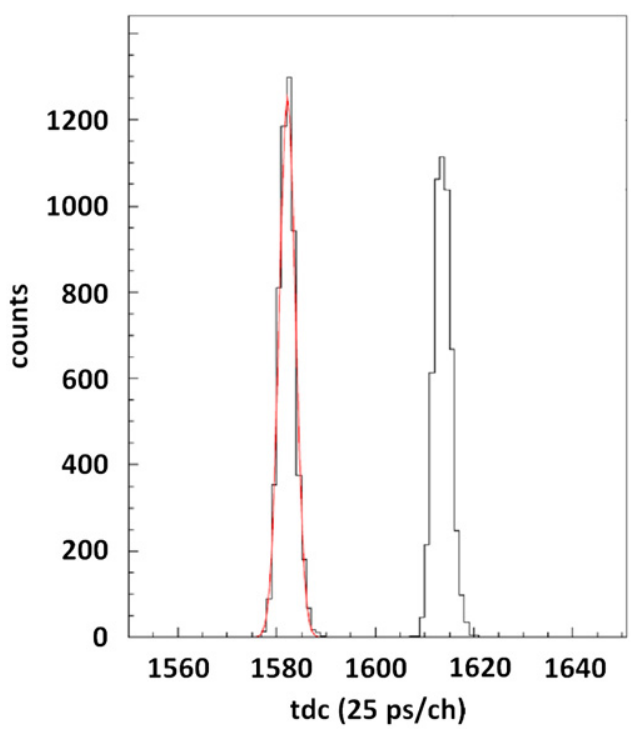

Fig. 7. Superimposed TDC spectra of the MPPC without (left) and with (right) preamplifier. The red superimposed line is the Gaussian fit of the peak. Time resolution ( $\simeq 30 \mathrm{ps}$ ) is not worsened by the pre-amplifier. (For interpretation of the references to color in this figure legend, the reader is referred to the web version of this article.)

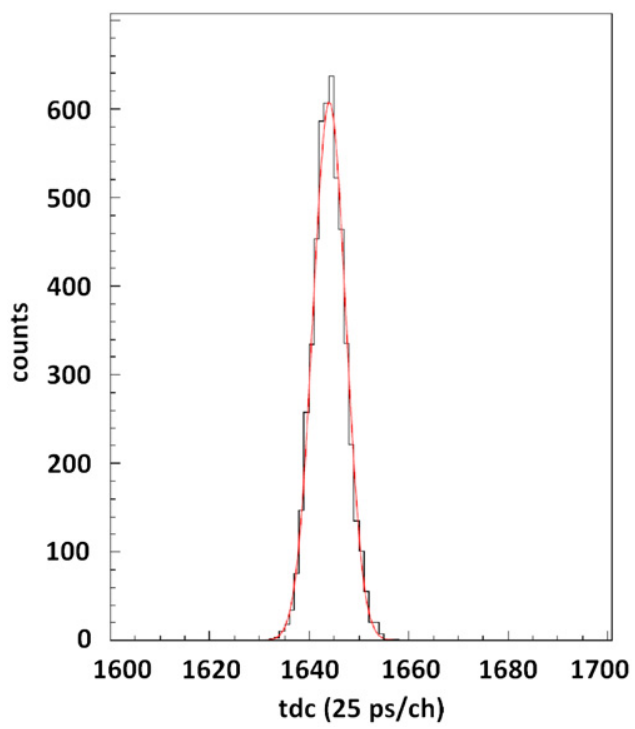

Fig. 8. TDC spectrum of the MPPC coupled to a scintillating fiber. The red superimposed line is the Gaussian fit of the peak. Time resolution ( $\simeq 80 \mathrm{ps})$ is worsened with respect to the one measured without the fiber. (For interpretation of the references to color in this figure legend, the reader is referred to the web version of this article.)

surface. The laser, triggerable with short pulses ( $\simeq 1 \mathrm{~ns}$ ), was externally driven by a pulse generator. The time difference between the trigger and the MPPC signals was collected both with and without the preamplifier. Results are shown in Fig. 7.
The measured time resolution $(\sigma)$ is $\simeq 30 \mathrm{ps}$. The same results were obtained both with and without the preamplifier. In order to investigate the jitter induced by the scintillating fiber, the same measurement was also performed with the laser pointed on the fiber and the obtained resolution is $\simeq 80$ ps (see Fig. 8).

The tests of the 64 channel trigger prototype for AMADEUS are presently underway, both in laboratory and on various beams $\left(\mathrm{e}^{-}\right.$, $\pi$ and $\mu$ at PSI $\pi \mathrm{M}-1$ beam and BTF facility at LNF-INFN).

\section{Conclusions}

The AMADEUS experiment has the goal to perform low-energy charged kaons interactions with nuclear matter measurements. In order to do this, a trigger system for the $\mathrm{K}^{+} \mathrm{K}^{-}$pairs delivered at DAФNE is needed, to be operated in the magnetic field of the KLOE detector. Two prototypes of the trigger system based on scintillating fibers read by MPPC were built and tested. Very promising results were obtained. With a first prototype, consisting of five Sci-Fi read at both ends by MPPCs, a time resolution of $300 \mathrm{ps}(\sigma)$ was obtained, and kaons were seen in a test measurement on DAФNE. A second prototype, with $32 \mathrm{Sci}-\mathrm{Fi}$ read by 64 MPPCs, with a new and more performant readout electronics was then built and characterized with a laser system in laboratory. The obtained result, a time resolution of $80 \mathrm{ps,}$ shows that this system is a valid candidate for the AMADEUS trigger. Further tests are presently underway, in order to have a more refined tuning of the parameters for the final system.

\section{Acknowledgments}

Part of this work was supported by the European CommunityResearch Infrastructure Integrating Activity "Study of Strongly Interacting Matter" (HadronPhysics2, Grant agreement no. 227431) under the Seventh Framework Programme of EU. We thank the DAФNE staff and to BTF-LNF team for the excellent working conditions and support. We thank as well Paolo Branchini and Roma 3 University for useful advices and help in performing the tests with the Laser system.

\section{References}

[1] The AMADEUS Collaboration, AMADEUS Phase-1: Physics, Setup and Roll-in Proposal LNF-07/24(IR), 2007.

[2] M. Adinolfi, et al., Nuclear Instruments and Methods in Physics Research Section A 461 (2001) (1-3) 25.

[3] D. Alesini, et al., DAФNE upgrade for SIDDHARTA RUN LNF-06/33 (IR), 2006.

[4] C. Milardi, DAФNE Interaction Regions Upgrade〈arXiv:0803.1450v1〉.

[5] Z. Sadygov, et al., Nuclear Instruments and Methods in Physics Research A 567 (2006) 70.

[6] N. Otte, SNIC Symposium, Stanford, California, 3-6 April 2006.

[7] M. Dinu, et al., Nuclear Instruments and Methods in Physics Research A 572 (2007) 422.

[8] M. McClish, et al., Nuclear Instruments and Methods in Physics Research A 572 (2007) 1065.

[9] A. Antonelli, et al., Nuclear Instruments and Methods in Physics Research A 370 (1996) 367.

[10] 〈www.detectors.saint-gobain.com〉.

[11] M. Bazzi, et al., Phys. Lett. B 681 (2009) 310. 